\title{
LAND DREAMING: IDENTIDADES Y ARRAIGO EN LA ESCRITURA DE SHARON BLACKIE Y PAT MORA ${ }^{1}$
}

\author{
Carmen García Navarro ${ }^{2}$
}

\begin{abstract}
Land dreaming: identidades y arraigo en la escritura de Sharon Blackie y Pat Mora
Resumen: Las obras de Sharon Blackie y Pat Mora enfatizan la necesidad de una mayor conexión de los individuos con el medio natural. Desde la necesidad personal de fortalecer los vínculos de pertenencia a un lugar y una comunidad, las dos autoras exploran sus raíces y el entramado de la historia personal. De la respuesta artística a esa necesidad surgen narrativas y poéticas que reconocen representaciones antiguas y actuales sobre el cuidado del planeta, y el de tradiciones, relatos y mitos de los lugares que habitamos.

Palabras clave: Construcción de identidades, tránsitos, arraigo, pertenencia, econarrativa, ecopoética.
\end{abstract}

\section{Land Dreaming: Identities and Belonging in Sharon Blackie's and Pat Mora's writing}

Abstract: The works of Sharon Blackie and Pat Mora emphasize the need for connection with the natural environment. Given the need to strengthen bonds of belonging to the community as key for the construction of identities, both writers explore their roots to produce narratives and poetics that name former and current representations of what unites us to this planet. Similarly, they argue about the relevancy of old and current representations, traditions, stories and myths of the places we inhabit.

Key words: Identity construction, transitions, settling, belonging, econarrative, ecopoetry.

\section{Introducción}

Me gustaría comenzar diciendo que al hablar de las escritoras Sharon Blackie (1958) y Pat Mora (1942) lo haré sobre una práctica repetida mil y una veces en la historia de la humanidad y en la historia particular de cada uno y cada una de nosotros: la exploración del sueño del arraigo y la formación de las identidades como fruto del diálogo entre culturas. Las dos autoras, Blackie y Mora, exploran sus raíces y el relato de la construcción del sí, buscando lo que une a la tierra y permite, además, nombrar

\footnotetext{
${ }^{1}$ Fecha de recepción: 20/11/2015.

Fecha de aceptación: 04/12/2015. ${ }^{2}$ Profesora Asociada, Departamento de Filología, Área de Filología Inglesa, Universidad de Almería; $\square$ mgn024@ual.es.
} 
representaciones antiguas y actuales sobre el cuidado del planeta, el conocimiento de las tradiciones, los paisajes, los relatos y los mitos de los lugares que habitamos.

Esta historia común, que me parece adecuada como punto de partida para mostrar las líneas maestras de la literatura de Blackie y sus conexiones con la obra de otras autoras, como Mora, refleja la preocupación por la deriva que ha tomado en distintas zonas del planeta el modo en que los individuos tratan y maltratan el medio natural ${ }^{3}$. No podemos dudar de que vivimos en mundo donde se imponen los usos de las tecnologías como mito, es decir, tomadas como referentes principales desde el punto de vista de la socialización, y donde, al mismo tiempo, las identidades se van reconstruyendo como procesos vivos en los que caben experiencias creativas que trascienden lo puramente individual para sentirse parte de la comunidad. Lo dicho se expresa en las obras de ficción de estas autoras mediante la construcción de un sí en procesos de tránsito, no sólo desde el punto de vista físico sino también emocional y social, que permite al sujeto situarse en una plataforma para contemplar lo que ha sido su vida y en lo que se ha transformado esa vida en el momento presente.

\section{Mujeres en tránsito}

Conocí a Sharon Blackie en 2012 y un año después pudimos reunirnos en su casa de la isla de Lewis, en las Hébridas Exteriores escocesas. El título de este trabajo nace de la entrevista que realicé a Blackie en 2012, tras haber conocido su trayectoria y parte de su obra. Consideré importante tener en cuenta la influencia de la poeta chicana Mora en su escritura, influencia reconocida y expresada por la propia Blackie en distintas oportunidades $^{4}$. La autora, inglesa descendiente de escoceses por parte de padre y de irlandeses por línea materna, viajera incansable y siempre en búsqueda de un lugar que

${ }^{3}$ Por ejemplo, Charlotte DuCann, Jay Griffiths, Mandy Haggith, Jane Hirschfield, Sara Maitland, Marylin Nelson, o Terry Tempest Williams, entre otras.

${ }^{4} \mathrm{~A}$ lo largo de estas páginas mostraré ejemplos de dicha influencia, así como la cita donde Blackie reconoce abiertamente esta influencia (véase nota 17). 
pudiera llamar propio, se había trasladado a la isla de Lewis con su marido. Allí continuaron dando vida a su proyecto editorial, Two Ravens Press, creado conjuntamente en 2006 y fundaron la revista EarthLines. A estos proyectos de trabajo se sumó el que mantiene la autora desde su página en internet. Su propósito era encontrar arraigo, como ciudadana y como escritora, y su deseo de involucrarse en la comunidad la llevó a hacerse copropietaria de una pequeña granja para cultivar la tierra y cuidar unos pocos animales 5 . En ningún caso era esto un intento de separarse de la comunidad. Muy al contrario, su propósito era formar parte de dicha comunidad e acogida asumiendo lo que tradicionalmente ha sido la principal actividad económica de los habitantes de la isla de Lewis. Participaría así, de manera sostenible, del cuidado de un medio natural como el de esas islas, sobre las que actualmente penden amenazas de destrucción del ecosistema y el paisaje ${ }^{6}$.

Me referiré primero a la experiencia personal y literaria de Blackie, quien, tras cinco años en la isla de Lewis, se trasladaría en 2014 a Donegal, en Irlanda. En los años vividos en Lewis, la escritura de Blackie se iba ensanchando, como un espacio acogedor de la necesidad de hallarse, para hallar sustrato que luego es creación y obra creativa. Como investigadora, surgían distintas preguntas al situarme ante la obra de Blackie, siendo una de ellas: ¿dónde estaba/está, entonces, el lugar llamado propio, la casa, el hogar, home, el signo que ilumina la señal de pertenencia? Según la autora, en los sucesivos desplazamientos a lo largo de su vida, su objetivo no ha sido buscar el lugar perfecto:

rather, at each of those different stages in my life it has seemed as if different places have been needed. And each of those different places -the purely physical aspects of these places, as well as the various and varying human cultures that I've found in them- have had different things to teach me. And so in each place I've lived, (...) I've found myself for the duration of that stay digging as deeply into it as I can. Because this is the only way in which I can learn the lessons of place, whether I'm inclined to a permanent rootedness or not. And so in my own life, as in my writing, I've come to believe that different landscapes produce different psychological states and so different transformations. For example, living in the bleak, wild, rocky

${ }^{5}$ En Escocia, croft es una pequeña parcela con fines de explotación familiar cuya propiedad se comparte con la comunidad.

${ }^{6}$ Proyectos, por ejemplo, como los del magnate Donald Trump de convertir un tercio de la isla en el mayor campo de golf de Europa (véase EarthLines, 2012). 
landscape of the Outer Hebrides taught me about extremity. About how far we can push ourselves, about resilience, about taking a stand. Moving to Donegal is in some way a return from that extremity, a need to reconnect with green and growing things again, and with community. (Blackie 2013) ${ }^{7}$

\section{Land dreaming: el sueño de un lugar propio}

Las palabras de Blackie reflejaban el enigma sobre el arraigo y la pertenencia, vivo como un organismo en proceso continuo de creación y evolución. Según Tim Ingold (2012), tal enigma es parte de la esencia de lo que significa habitar nuestro planeta, que significa "to be embarked upon a movement along a way of life" ${ }^{8}$. Blackie no se siente inglesa ni escocesa, o se siente de diferentes lugares y de ninguno particular. La autora afirma, citando a Stuart Hall, que "That's exactly the diasporic experience, far away enough to experience the sense of exile and loss, close enough to understand the enigma of an always postponed 'arrival'” (Blackie 2013) ${ }^{9}$. Llegados aquí, surgía otra pregunta, creo que lógica: ¿quedará todo esto simplemente silenciado, callado, como tantas veces han quedado en el silencio o en la invisibilidad las voces de otras autoras? Porque, una vez más, el texto creativo se presentaba como espacio-mediación entre la creación y la vida, en este caso de mujeres que viven a diario su realidad como mujeres y escritoras, recreando un orden desde el deseo de poner claridad en las coordenadas que las vinculan a una autoridad que las coloca en condiciones para dirigir su trayectoria vital hacia un horizonte de libertad creativa, personal y colectiva, incorporando las dudas o las

\footnotetext{
7“ $\mathrm{Al}$ contrario, en cada etapa de mi vida he necesitado un lugar distinto. $\mathrm{Y}$ cada uno de esos lugares -los aspectos puramente físicos, así como la variedad de personas y culturas que he encontrado en ellos- me han enseñado cosas diferentes. En cada uno de los lugares en los que he vivido me he involucrado a fondo mientras he vivido allí. Porque esa es la única manera en la que puedo aprender de lo que un lugar tiene que enseñarme, tanto si me he sentido arraigada a él de manera permanente como si no. Y así, como sucede en mi vida, y en mi escritura, creo que los lugares y los paisajes producen estados psicológicos distintos y distintas transformaciones. Por ejemplo, vivir en un medio desolado, rocoso y salvaje como el de las islas Hébridas Exteriores me enseñó sobre lo extremo. Sobre hasta dónde somos capaces de llegar, sobre la resiliencia y la resistencia. Mudarnos a Donegal es, de alguna manera, un regreso desde lo extremo, una necesidad de reconectar con lo fértil, y con la comunidad." La traducción de esta cita y las siguientes es mía y debe entenderse como una traducción libre y no especializada.

8"Estar inmerso en el movimiento a lo largo de la vida."

${ }^{9}$ "Esa es exactamente la experiencia de la diáspora, suficientemente lejos de la vivencia del exilio y la pérdida, pero cercana como para entender el misterio de una "llegada" siempre postpuesta".
} 
contradicciones como algo consustancial a la vida de las personas, una escritura desde donde se indaga en el reconocimiento del origen, acción que exige abrirse a lo otro que hay más allá de sí, para lo cual ha de asumirse la práctica de la relación en el espacio público pero también con y desde el medio natural, tantas veces humanizado, espacios ambos abiertos, diversos, plurales, y donde se manifiestan el desorden y lo imprevisible. La ausencia de neutralidad, pues, alimenta el sustrato sobre el que se asienta al menos una parte, si bien una parte significativa, de la producción literaria de autoras como Blackie, al igual que sucede con Mora, de la que hablaré un poco más adelante.

Entonces, hablamos de un espacio en el que arraigarse, donde hay un yo que se aquieta en tierra, y un espacio para la comunidad y para el otro o la otra. En la ficción de Blackie, Cat, la protagonista de la novela The Long Delirious Burning Blue (2008) se expone al hallarse a uno y otro lado de un espacio fronterizo, del límite, del margen. Con cuarenta años recién cumplidos, el proceso de creación de su identidad se ve condicionado por una infancia con un padre ausente, una madre alcohólica y una tía-abuela que la ayuda a pisar tierra firme cuando Cat decide transitar la senda del reconocimiento de sí, su historia propia. El arraigo no puede darse en ninguno de los lugares donde ha vivido. Esa imposibilidad es también home: el lugar de donde hay que irse para que se den otros comienzos, por ejemplo darse voz a sí misma. Quizá más tarde el sujeto pueda regresar. Ese lugar-noción adquiere un máximo sentido cuando se transforma en pérdida y en desconexión, en algo que ha quedado atrás y que el individuo desea y reclama, como la propia identidad. La frontera se disuelve en el reconocimiento de que el anonimato es suplantado por la identidad reconocida en la esfera pública, en el conocimiento del habla local, las tradiciones, los relatos del lugar. El sujeto ha perdido la casa, el hogar, el sitio donde sentirse arraigado. Lleva consigo el cansancio de verse entre espacios físicos y simbólicos limítrofes, obligada a desplazar la mirada a un lugar y a otro continuamente (Augé 1996: 97), a reencontrarse a diario en la necesidad de una toma de postura que permite el movimiento de desplazamiento hacia el encuentro con el origen:

It has always seemed to her that shorelines are magical places: anything can happen here in this border land between water and earth -this fluid border that shifts with 
the tides, in perpetual motion and flow. At places like this you can cross into the Other world-or Fairyland, as they call it in some stories. [...] All of them thresholds between one world and another. (Blackie 2008: 126-127) ${ }^{10}$

Home, pues, es también la intimidad de una tierra que recibe y permite conocerla o reconocerla, una identidad en movimiento que ya es. Es el sueño sobre el lugar, sobre la tierra como hogar propio. Y ahí adquiere máximo valor el cuidado de ese lugar, pues el lugar nos conforma, como dice Blackie, nos permite identificarnos con él, un individuo que lleva tras de sí un conjunto de formas de ser dispersas que ahora quieren hacerse identidad propia. Desde luego, Cat debe vivir determinadas vivencias una vez llegada a la ciudad de Phoenix, bajo la luz del desierto de Sonora en Arizona, a donde se ha trasladado por trabajo. Entonces, el recuerdo, las voces de las mujeres de su vida, el divorcio de su primer marido y la experiencia de nuevas relaciones con distintas personas, la hacen cuestionarse si home es un espacio físico, una lengua, un espacio emocional, o un espacio histórico. Estas preguntas de Cat contienen, a mi modo de ver, una intención política. Están formuladas por un sujeto femenino en una posición de vaivén entre la dependencia y la independencia, la actividad y la pasividad que históricamente ha caracterizado a los modos y posibilidades de estar en el mundo para muchas mujeres, y de este movimiento de ida y vuelta del ser mujeres forma parte una necesidad que no es posible traducir única ni completamente mediante las estructuras del lenguaje ni mediante estructuras de representación y significación científica y teórica, debido a la unicidad y la singularidad de la persona, singularidad que conlleva la formación de la identidad propia.

\section{Habitar la frontera y su espacio}

Llegada a este punto, Cat ya no es una viajera, sino la habitante de una realidad de tránsito y también de una intersección, donde pesa mucho la pregunta sobre qué es estar

\footnotetext{
10"Siempre le ha parecido que las orillas son lugares mágicos: en esta tierra fronteriza puede ocurrir cualquier cosa -esta frontera fluida que se cambia con las mares, en perpetua fluidez y movimiento. En lugares como estos puedes cruzar al Otro mundo o al País de las Hadas, como lo llaman en algunos cuentos. (...) Todos son umbrales entre un mundo y otro."
} 
en casa. Incluso la lengua limita o expande las posibilidades de los significados, sentirse en casa o no, aunque se trate de la lengua inglesa hablada a uno y otro lado del Atlántico. Sabemos que las fronteras están habitadas por migrantes, desplazados que, según dónde nos coloquemos, visibilizan el límite, el espacio fronterizo. La lengua común no puede impedir ocasiones de visibilización de la existencia de fronteras. Así, dialogando con un compañero de trabajo, Cat se ve nuevamente confrontada:

-"One of these days I"m going to teach you to speak the language properly. If you say "mad" over here it means "angry". You mean "crazy."

I roll my eyes. 'Americans. After ten years I still can't understand you.'” (Blackie: 2008: 100$)^{11}$

Esta disociación está también presente en la poesía de una de las autoras de referencia de Blackie, Pat Mora: “I love Pat Mora's work, and yes, she's always been a big influence on me. There is something in her writing which is more than just writing about place -it's about an intense identification with place, expressing all of the ways in which place can make us what we are" (Blackie 2013) ${ }^{12}$. Desde su infancia, Mora ha experimentado la condición de fronteriza al ser oriunda de El Paso, enclavado en el desierto mexicano, en el sur rural de Texas. La lengua española fue la lengua de su educación familiar y la lengua inglesa la su educación formal. Este hecho ha marcado su trayectoria como mujer y como creadora, llena de desafíos y no exenta de contradicciones $^{13}$. Una parte de la producción poética de Mora está escrita en español y otra en inglés. En los poemarios Chants (1984), Borders (1986) y Communion (1991) la autora explota al máximo las posibilidades de esa mezcla, utilizando términos en castellano y en inglés, así como introduciendo palabras del argot latino, del afroamericano

\footnotetext{
11“'Un día te voy a enseñar a hablar bien. Si dices 'mad' aquí significa 'enfadada'. Lo que quieres decir es 'loca'. Aparto la mirada. 'Americanos. Después de diez años aquí todavía no os entiendo."

12“"Me interesa la obra de Pat Mora, y sí, siempre me ha influido mucho. Hay algo en su escritura que va más allá de la narración sobre un lugar: es una profunda identificación con el lugar, que habla de todas las maneras en las que el lugar puede hacernos ser quienes somos."

${ }^{13}$ Por ejemplo, la necesidad de publicar en inglés. En otro lugar he tratado este asunto de manera más extensa, véase García Navarro 2007.
} 
y del Spanglish. Diversas escuelas han enfatizado la lectura de textos de ficción escritos por mujeres como forma de oponer resistencia a la ideologización (Suárez Briones, Martín Lucas y Fariña Busto 2000: 10). Dichos textos habrían suplantado la representación del margen, yendo más allá del mero encasillamiento en hipotéticos grupos o subgrupos, para hacerse voz de mujeres que representan a sujetos complejos, no uniformes, que aprenden, desaprenden y acogen sus peripecias vitales desde una óptica imposible de ceñir a un único patrón. La lengua es lugar de encuentro, puente que da sentido estético y ético a la propuesta artística. Es canal entre el espacio interior y el espacio público, llevando el deseo de las creadoras de nombrar el mundo a partir de sí y de la llamada a la relación. Cualquiera que sea su procedencia y su punto de destino, estas autoras migrantes tratan continuamente de negociar el valor adquirido de la pertenencia a un ámbito distinto al de su procedencia.

\section{La práctica de la mezcla entre culturas}

Desde lo dicho anteriormente, comparto igualmente las afirmaciones de Carol Gilligan recogidas por Joe L. Kinchloe y Shirley R. Steinberg sobre la reducción a la que se someten actuaciones y razonamientos de numerosas mujeres por el hecho de que en sus experiencias intervengan valores y creencias en los que la subjetividad ocupa un espacio propio (Kinchloe y Steinberg 1999: 188). Lo digo porque la poesía de Mora no oculta la fascinación que produce en los sentidos y en la cognición del sujeto femenino el deseo de sentirse parte del medio natural. Mora alude repetidamente en sus obras a las mujeres que recurren a saberes no exclusivamente racionales para ayudarse o inspirarse en distintas situaciones de peligro, enfermedad y sufrimiento padecidos por ellas o por miembros de la comunidad, o a la forma de entender la sexualidad y la relación con el ser amado. El poema "Love Ritual" es un ejemplo de lo anterior. La primera parte cuenta la manera en que los mexicanos celebran el rito fúnebre: se llevan a la mesa velas, mole picante cocinado por las mujeres, tequila, pan dulce y mangos para convocar a quien acaba de fallecer. En la segunda parte de la composición, el yo lírico irrumpe 
anteponiendo el ansia de realización de un deseo propio mediante la consecución de otros rituales: "Outside my door I'll sprinkle yellow/ flower petals. Carefully I'll place/ my picture, the poem I wrote you,/ a sketch of two lovers removing/ each other's clothes. I'll light/ green votives, and you'll be pulled/ back too. And maybe stay" (Mora 1984: 27) ${ }^{14}$. El recurso a este conocimiento subordinado de las mujeres ${ }^{15}$ centra la atención en la capacidad de las mujeres para relacionarse con su cuerpo, con el medio natural del que se sienten parte, y en la manera de entender la afectividad. Aquí, la ausencia de neutralidad alimenta el sustrato sobre el que se asienta una parte de la producción literaria de Mora. El diálogo y la mezcla entre culturas permite un desmantelamiento del canon literario e ideológico, da frutos jugosos por cuanto que posibilita un acercamiento a múltiples perspectivas, formas diversas de mirar el mundo, con ojos que nos hacen progresivamente "conscientes de nuestros puntos ciegos, de la presencia de los diferentes otros y de la dificultad de ver su perspectiva particular" (Carrera Suárez, en Suárez Briones, Martín Lucas y Fariña Busto 2000: 79).

La tierra y sus criaturas, y la necesidad de atención y cuidado que estas requieren, un cuidado vigilante que ha formado parte de los procesos de vida de generaciones y que habla hoy de un vínculo necesario, así como de una autoridad moral y espiritual de los habitantes de esos lugares. En este sentido, la presión ejercida por el ser humano sobre el medio natural es un componente esencial de la narrativa de Blackie. La modernidad ha hecho esfuerzos por crear un mundo mejor que el conocido hasta ahora por otras generaciones anteriores a las nuestras, pero esa misma modernidad ha abusado y abusa del medio natural hasta un extremo que muy pocos de nuestros antepasados hubieran podido imaginar. La sobreexplotación de los recursos y el descuido del medio ambiente, así como el abandono de diversas áreas naturales de nuestros entornos, no es sino una forma más de violencia, que se traduce como violencia contra nuestro planeta y, al fin, contra el propio ser humano. Todo ello ha conducido al ser humano a un desarraigo de tipo íntimo que evidencia la existencia de pérdidas de valor ecológico pero también

\footnotetext{
14“"Esparciré pétalos/amarillos en la puerta de mi casa./Colocaré con cuidado mi foto/y el poema que te escribí,/el dibujo de dos amantes desnudándose./Haré una ofrenda/con velas de color verde y así te haré volver/a ti también. Y quizás te quedes."

${ }^{15}$ Estoy empleando aquí el término de Kinchloe y Steinberg 1999: 203.
} 
personal. Cuidar el medio natural es reconocer como parte de sí la tierra que acoge al individuo, que le proporciona alimento y cobijo. En este sentido, Sir John Lister-Kaye habla de una necesidad de encuentro, el del individuo con el medio natural, como una noción que en lengua inglesa queda recogida en el término wild: "wild the notion, wild the unruly concept has always been with us, dogging our heels ever since we were truly wild ourselves" (Lister-Kaye 2013: 5) ${ }^{16}$. Se trata de un término que evoca mucho más que nuestros orígenes, pues se diría compuesto por multitud de caras y aspectos: sentimientos, impulsos, sueños, emociones, deseos, miedos. Hace referencia a la vida y la muerte, a los ciclos eternos de la naturaleza. Nombra lo que se tiene por excéntrico, con un matiz que alude al gusto por el riesgo. Igualmente, se asocia con todo lo que no podemos controlar: wildlife, wildcat, por citar sólo unos ejemplos. Y añade Lister-Kaye: "Wild is (...) the adjective that has mapped our wrench away from nature's grip, and the word we fall back on when something or someone doesn't quite fit the manageable and domesticated, manseparated-from-nature mould" (Lester-Kay 2013: 5) ${ }^{17}$. Llegados aquí, recordamos que Henry David Thoreau se refirió a esta noción aludiendo, principalmente, al sentido de no domesticación de la voluntad de un ser sobre otro. Para Thoreau, wildness ${ }^{18}$ se refiere a una cualidad o un estado, y wilderness ${ }^{19}$ a un espacio natural. De manera similar a Thoreau, otros autores han escrito sobre la necesidad de pensar y repensar nuestros orígenes con relación al medio en que habitamos, respetar los ritmos de la naturaleza, integrándolos como parte esencial de la vida en este planeta, no como fuerzas que hay que conquistar y explotar eternamente. Así se expresan John Clare ${ }^{20}$, John Muir (en Lister-Kaye 2013:7) ${ }^{21}$ o Jay Griffiths (2012) cuando habla de que wild o wildness existe como "a peculiar tilt of the will" 22 .

\footnotetext{
16“'Salvaje, como noción, como concepto de lo rebelde, ha estado siempre entre nosotros, persiguiéndonos los talones desde que éramos realmente salvajes."

17،'Salvaje es el original ellos y nosotros. Es el adjetivo que ha marcado el modo en que hemos dado la espalda a nuestra comprensión de la naturaleza, y es la palabra a la que recurrimos cuando algo o alguien no encaja dentro del molde de lo manipulable, de lo domesticado, del-hombre-separado-de-la-naturaleza." ${ }^{18}$ Estado salvaje; asilvestrado. El diccionario Collins contempla también el sentido de extravagante.

${ }^{19}$ Tierra salvaje, naturaleza. El diccionario Collins se refiere, además, al sentido figurado del término: estar al margen.

${ }^{20}$ Véase Acroyd 2012: 42 y Canton 2012: 13.

${ }^{21}$ Véase Acroyd 2012: 42 y Canton 2012:13.

22"Una inclinación especial de la voluntad."
} 
Todo lo anterior, junto con su compromiso personal por vivir de manera coherente con los ritmos de la tierra y la naturaleza, dedicando para ello una parte significativa de su tiempo a la explotación de los recursos únicamente a la escala de una economía de subsistencia de tipo familiar, y con el uso muy racionalizado de la tecnología, ha estimulado la mirada crítica de Blackie hacia la realidad de un mundo como el nuestro, donde convergen alicientes de corte ultramoderno con valores distintos en los usos del tiempo, los bienes de consumo y las tecnologías o la relación con el medio natural. Con una escritura permeable a las mezclas de la vida real y lo ficticio, Blackie busca alternativas discursivas para expresar sus intereses narrativos, aprovechando el legado de escritores y escritoras como los mencionados. Para Blackie, wild es reconocer nuestra humanidad en sus más básicas necesidades, sin aderezarlas con creencias o prácticas que se nos ofrecen, hoy en día, como mito, como por ejemplo la utilización de las tecnologías como señas de identidad de las sociedades occidentales en la actualidad. La suya es una vinculación cuidadosa con el entorno, coincidiendo así con otras voces contemporáneas, como la de Griffiths, cuando afirman que "it is part of our very humanity to care about wildness, animals, landscapes and freedom" (Griffiths 2012a:26) ${ }^{23}$.

Home es el deseo y es también el rechazo, como se desea el núcleo materno del cual provenimos y se rechaza la parte de impostura para ser uno mismo. El deseo de ser y ser en un lugar al que se pueda llamar home se experimenta también en el cuerpo toda vez que ha sido reconocido como el depositario de la parte de sí que acepta, que reconoce el origen y todo lo que uno ha sido. Así sucede con Cat en Arizona. Cat llega a la ciudad de Phoenix después de haber vivido en Europa, América y Japón, siguiendo siempre el impulso de huir de las dificultades y miedos que la han traído desde su niñez y su juventud hasta esta ciudad surgida en pleno desierto.

Según cuenta Blackie a lo largo de nuestra entrevista, los lugares y los paisajes tienen una cualidad arquetípica que, en este caso, sirve a la autora para hacerlos parte activa y protagonista de su escritura. Y algunos lugares ofrecen una cualidad distintiva que posibilita el que se produzcan cambios o el comienzo de algunos cambios,

\footnotetext{
23،'Una parte de nuestra humanidad consiste en cuidar del medio natural, de los animales, de los paisajes y de la libertad."
} 
transformaciones que nos abren a la sorpresa. Los lugares reflejan lo que ocurre en las vidas y en los pensamientos de los personajes y aquellos con los que se relacionan e identifican. Suministran información sobre la naturaleza del personaje y de su propia peripecia vital. Aportan pistas sobre ciertas actitudes y decisiones de los personajes, como sucede a Cat con el desierto. Así lo explica Blackie: "in some way the stark openness of the desert, when [Cat] finally emerges from the safety of the city of Phoenix (...) allows herself to confront it, requires those changes of her" (Blackie 2013) ${ }^{24}$. Y seguidamente dejamos hablar a Cat: "this desert provides no such respite. Out there, there is nowhere to hide. The sun shines down on you fiercely, illuminating all your hollowed-out emptiness, casting far too much light on your daily fumblings for adequacy" (Blackie 2008: 241$)^{25}$.

A mi modo de ver, Cat se ve impelida a desmontar el edificio de su pasado para poder narrar el relato de ese pasado de otra manera. Sacar lo que no sirve. Y el desierto es un medio que la coloca en esa disposición porque es pura claridad, y simplicidad: "the vast and arid emptiness cuts through the clutter of your tiny, everyday thoughts" (Blackie 2008: 241$)^{26}$. Si en Escocia encontró a las ancianas contadoras de relatos sobre el lugar y sus habitantes, en el desierto conocerá a otra mujer de avanzada edad, María Mercedes, a la que escuchará contar otros relatos, los de este lado, el del desierto, y de formas nuevas de hacerse cargo de uno mismo y del medio que habita.

Poco a poco, Cat va reconociendo por sí misma el valor de la sencillez en los encuentros con distintas personas, el afrontamiento de distintas realidades de su vida, contribuyen a ese nuevo encuentro consigo y con lo que le rodea. El desierto tiene aquí su papel para enseñar sobre la inteligencia de sus pobladores, el canto del fuego, de la luz y del agua. En la puerta de la casa de María Mercedes, un conjunto de altos saguaros ${ }^{27}$ la

\footnotetext{
24 "Un espacio completamente abierto como el desierto, de alguna manera permite a Cat, cuando esta finalmente sale de una ciudad como Phoenix, que percibe como un medio seguro, confrontar esa seguridad; aquel medio demanda de ella esa transformación."

${ }^{25}$ "Este desierto no da tregua. No hay donde esconderse. El sol brilla sobre ti con furia, iluminando todo tu vacío, arrojando luz sobre el torpe intento diario de sentirte adecuada."

26“"El enorme y árido vacío se cuela hasta el desorden de tus diminutos pensamientos."

${ }^{27}$ El saguaro (Carnegiea gigantea) es el cactus más abundante y típico del desierto de Sonora, Arizona. Puede llegar a medir hasta 12 metros de altura. Necesita condiciones óptimas de luz y sequedad para alcanzar su característico porte. Crece en compañía de pequeños árboles y arbustos, cuyas raíces protegen
} 
reciben: "This magic of the desert: elemental. Stripping you down to the very essentials. Stripping you bare. All the way down to the bones" (Blackie 2008: 329) ${ }^{28}$. Y en la voz de Blackie, "the places where [the characters] find themselves reflect the psychological processes of these characters. They're moving on not just physically, but psychologically. Finding not just places that they can relate to, but the places that will create the necessary transformations in them" (Blackie 2013) ${ }^{29}$. En algunos lugares, el cambio vivido por el individuo está, de alguna manera, facilitado, o propiciado, por las características de ese lugar. En este sentido, la vida de Blackie como mujer y escritora en las Hébridas Exteriores ha sido como la vida en el desierto. Esto puede resultar paradójico porque en las islas escocesas el clima es muy húmedo y el del desierto es seco, pero ambos medios comparten aspectos de desolación y simplicidad, "a quality of 'no frills' ... and for sure I believe that places like that, where the landscape is extreme, lacking in softness, throw you back on your own internal resources in a way that other landscapes don't. You let them change you, you move with them, or you break yourself against them" (Blackie $2013)^{30}$.

Lo anterior queda recogido también en los versos de un poema de Mora como "Desert Women", del que Blackie se hace eco al hablar de la huella de la autora chicana en su escritura: "Desert women know/ about survival. Fierce heat/ and cold have burned our skin./ Like cactus, we've learned/ to pull in tender leaves. [...] But when we flower, we stun" (Mora, en Blackie, 2013) ${ }^{31}$. Desde luego, diferentes autoras han escrito sobre esto antes que Blackie y otras lo hacen hoy en día, decididas a poner en valor la ligazon possible del ser humano con el medio natural y el medio ambiente, y han centrado una

a las del saguaro cuando éste es muy joven para evitar que sean devoradas por roedores y otros animales. Sus flores, frutos y semillas son comestibles. Es una planta muy sensible a los cambios climáticos y al daño ambiental en general.

28"La magia del desierto: elemental. Te deja con lo esencial. Te desnuda. Te deja en los huesos."

29“'Los lugares donde se encuentran estos personajes reflejan los procesos psicológicos que están viviendo. Son personas en movimiento, no sólo física sino psicológicamente. Desean encontrar lugares con los que identificarse y donde puedan crear las condiciones necesarias para su transformación personal."

30"Una cualidad que no admite florituras. Y creo que paisajes extremos y duros como esos, te hacen mirar hacia tus propios recursos de un modo distinto al de otros. Les permites que te transformen, te mueves con ellos o te estrellas contra ellos."

31 "Las mujeres del desierto saben/de supervivencia. El calor/y el frío intensos han quemado nuestra piel./Como los cactus, hemos aprendido/ a llevar hacia dentro las hojas tiernas ... Pero cuando florecemos, asombramos." 
parte de su producción literaria en el desierto también, ${ }^{32}$ aunque, no duda en añadir: "When I look at writing by women which falls into the general category of "nature writing", what I see in the best of those writers is precisely this kind of intense identification with the landscape. You rarely see that kind of direct, deep, sensory connection in the writing of men" (Blackie 2013) ${ }^{33}$.

\section{Comentarios finales}

La escritura de Blackie indaga en nuevas formas narrativas, frutos de la observación, que expresan la subjetividad, especialmente la de las mujeres creadoras, y que superan el extremo puramente emocional, para centrarse en una aceptación de lo que es natural y no idealizado en lo que sucede alrededor. El texto es un espacio de diálogo donde la mezcla y el tránsito entre los espacios fronterizos permiten un desmantelamiento del canon literario e ideológico porque propician un acercamiento a múltiples voces y perspectivas.

A su vez, podríamos hablar de un rico tejido elaborado por escritoras con orientaciones diferenciadas pero que comparten trazos para cartografiar los espacios intermedios, los lugares-entre, los dos lados de las fronteras. Autoras como Blackie y Mora hacen suyo un espacio como el desierto de Arizona, que les sirve como fuerza inspiradora y nutritiva para los sentidos y se refleja en la dirección y evolución de sus narrativas. La escritura es, pues, instrumento mediante el cual se reclama un territorio propio. A través de ella se recrea el lugar home donde sentirse arraigado y prenda el proceso de evolución de las identidades. Donde sólo había anomia y desafección de sí y del medio, hay desarrollos de formas de conexión, de conocimiento para mujeres que no necesariamente sostienen el orden cultural dominante y que, sea cual sea el modo en que

\footnotetext{
${ }^{32}$ Véase nota 1. Sin duda, contamos también con la aportación de autores como Mark Cocker, Jeremy Hooker, Robert MacFarlane o Martin Shaw.

33“"Cuando leo narrativa de mujeres conocida como 'ecoliteratura' lo que observo en lo mejor de esa escritura es, precisamente, este tipo de gran identificación con el medio natural. Raras veces vemos este tipo de conexión directa, profunda, sensorial, en los escritores."
} 
eligen vivir, eligen hacerlo de un modo particular: proporcionándose una fuerte conexión con la tierra y con los seres vivos que la habitan, conociendo el lugar donde viven (conoce sus características geográficas, su ecología, su historia, sus tradiciones), y viviendo ese arraigo desde la responsabilidad por el medio y el hábitat. Conforman trayectorias personalísimas, que no dejan de ser colaborativas en proyectos de índole no sólo personal sino también comunitaria. Todo ello nos comunica la creación de discursos en los que la acción de recordar, la de conocer el presente y la de poner palabras a los hechos que acontecen, convierte esos discursos narrativos en acontecimientos y no en actividades apegadas a modas diversas o en meras anécdotas.

\section{Referencias bibliográficas}

Acroyd, Carry. 2012. "Changing Views. John Clare and learning to see". EarthLines. November 2012, issue 3: 42.45.

Augé, Marc. 1996. Los “no lugares”. Espacios del anonimato. Una antropología de la sobremodernidad. Barcelona. Gedisa.

Blackie, Sharon. 2008. The Long Delirious Burning Blue. Ullapool: Two Ravens Press.

Canton, James. 2012. “Untangling British Nature Writing”. EarthLines, May 2012, issue: 11-15.

Du Cann, Charlotte. 2013. "Holding a Door Open for the Ancestors". EarthLines, February 2013, issue 4: 56-58.

García Navarro, Carmen. 2007. "La frontera difusa. El cuerpo deseado y el cuerpo desterrado en la poesía de Pat Mora". Confluencia. Revista Hispánica de literatura y cultura. Spring 2007, vol. 22, $\mathrm{n}^{\circ} 2$ : 50-61.

Griffiths, Jay. 2012a "An Interview with Jay Grifiths”. EarthLines, May 2012, issue 1: 26-28.

Griffiths, Jay. 2012b. Wild. An Elemental Journey. London: Penguin Books.

2013. Entrevista a Sharon Blackie. Inédito.

Ingold, Tim.2012. "Looking for Lines in Nature". EarthLines, November 2012, issue 3: 48- 51 
Kinchloe, Joe L. y Shirley Steinberg. 1999. Repensar el multiculturalismo. Barcelona: Octaedro.

Lister-Kaye, John. 2013. “The quest for Wildness". EarthLines, February 2013, issue 4: 4-7.

Mora, Pat. 1984. Chants. Houston, Texas: Arte Público Press.

Stanford Friedman, Susan. 2004. "Bodies on the Move: A Poetics of Home and Disaspora". Tulsa Studies in Women's Literature, vol. 23, nº 2, Fall: 189-212.

Suárez Briones, Beatriz, Ma Belén Martín Lucas y Ma Jesús Fariña Busto (eds.). 2000. Escribir en femenino. Poéticas y políticas. Barcelona. Icaria. 